\title{
Optimizing the Detection of Subtle Insular Lesions on MRI When Insular Epilepsy Is Suspected
}

\author{
(D). Blustajn, DS. Krystal, (DD. Taussig, (DS. Ferrand-Sorbets, DG. Dorfmüller, and DM. Fohlen
}

\begin{abstract}
SUMMARY: Insular epilepsy is underdiagnosed and accounts for a number of failed operations. Identifying insular target lesions on MR imaging can help guide intracranial electroencephalography and improve the outcome of surgery. In this study, we present a novel method of exploring the insular region for subtle lesions on 3D MR imaging by MPR postprocessing of slices in oblique reference planes. Using this method, we retrospectively reviewed presurgical MRIs that were initially considered to have normal findings in 7 pediatric patients with intractable insular epilepsy. Insular epilepsy was confirmed in these patients on stereo-electroencephalography and histopathology. The MPR postprocessing method we describe helped detect subtle insular lesions in all 7 patients.
\end{abstract}

ABBREVIATIONS: $\quad$ FCD $=$ focal cortical dysplasia; SEEG = stereo-electroencephalography

$\mathbf{R}$ efractory epilepsy of the insula is one of the most challenging forms of epilepsy to diagnose and treat for a number of reasons. Due to the position of the insula situated at the crossroads of several lobes, the clinical semiology of insular seizures often orients toward a frontal, parietal, or temporal lobe origin. ${ }^{1}$ Furthermore, because the insula is covered by the opercular folds, its electrical activity is partly shielded from scalp electroencephalography. Several authors have shown that the ictal discharge in insular seizures could mimic frontal, temporal, central, or parietal seizure-onset zones, misleading the diagnosis. ${ }^{1-3}$

When insular epilepsy is suspected, stereo-electroencephalography (SEEG) is often required to accurately locate the ictal onset zone. ${ }^{1,4}$ The proper positioning of these intracranial electrodes depends on the ability of MR imaging to define a target lesion. When MR imaging is unable to define a lesion, the chances of a successful operation are significantly reduced.

Because the insula covers a small surface area of the brain and its cortex forms discrete gyri oriented in an oblique sagittal plane, it may be difficult to identify its architecture and its limits on MR imaging viewed in standard orthogonal planes (sagittal, axial, and coronal).

In this retrospective study of 7 pediatric patients who under-

Received April 3, 2019; accepted after revision June 17.

From the Diagnostic Neuroradiology Department (J.B., S.K.) and Pediatric Neurosurgery Department (D.T., S.F.-S., G.D., M.F.), Rothschild Foundation Hospital, Paris, France.

Please address correspondence to Martine Fohlen, MD, Pediatric Neurosurgery Department, Fondation Adolphe de Rothschild, 29 rue Manin, 75019, Paris, France; e-mail: mfohlen@for.paris; @FondARothschild

http://dx.doi.org/10.3174/ajnr.A6143 went an operation for refractory insular epilepsy, we show how MPR postprocessing of high-resolution 3D-T1WI and 3D-T2WI can help identify subtle insular lesions that were initially missed on presurgical MR imaging but were subsequently identified on SEEG and histopathology.

\section{METHODOLOGY}

Local regional ethics approval was obtained for this retrospective study.

\section{Case Selection}

Of the 293 patients who had undergone focal resection for refractory epilepsy in our institution between January 2009 and December 2016, we selected those with insular epilepsy on the basis of SEEG results (30 patients; 10\%). Of these patients, we excluded those MRIs positive for insular lesions (11 patients). Among the remaining cases, apparently "nonlesional," we excluded those whose presurgical MRIs were performed with insufficient resolution (maximum voxel size of $1 \mathrm{~mm}^{3}$ ) (12 patients).

Seven patients were retained in our study, 4 boys and 3 girls with a mean age at the time of presurgical MR imaging of 8.6 years (range, 3-14.7 years).

\section{Imaging Acquisition and Analysis}

MR imaging was performed on a $3 \mathrm{~T}$ Ingenia scanner (Philips Healthcare, Best, the Netherlands) using a 32-channel head coil. All of the scans were obtained with the patient under general anesthesia, as is routine in our institution for children younger than 7 years of age or for patients with difficulty in remaining calm for the duration of the examination. 


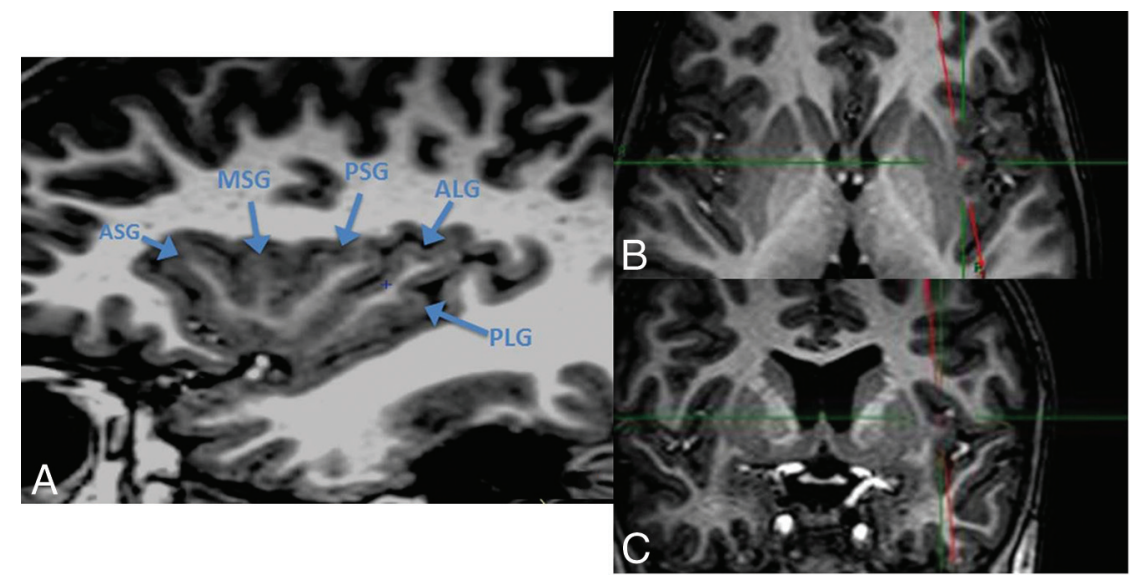

FIG 1. 3D-T1-weighted sequence with MPR processing showing the common gyral pattern of the insula: reference view to analyze the insula. $A$, TIWI oblique sagittal view, parallel to the base of the insula, anterior lobule. ASG indicates anterior short gyrus; MSG, middle short gyrus; PSG, posterior short gyrus. Posterior lobule: ALG indicates anterior long gyrus; PLG, posterior long gyrus, which appears classically shorter than the ALG. B, TIWI axial view shows the oblique cut plane parallel to the base of the insula. C, TIWI coronal view shows the oblique cut plane parallel to the base of the insula.

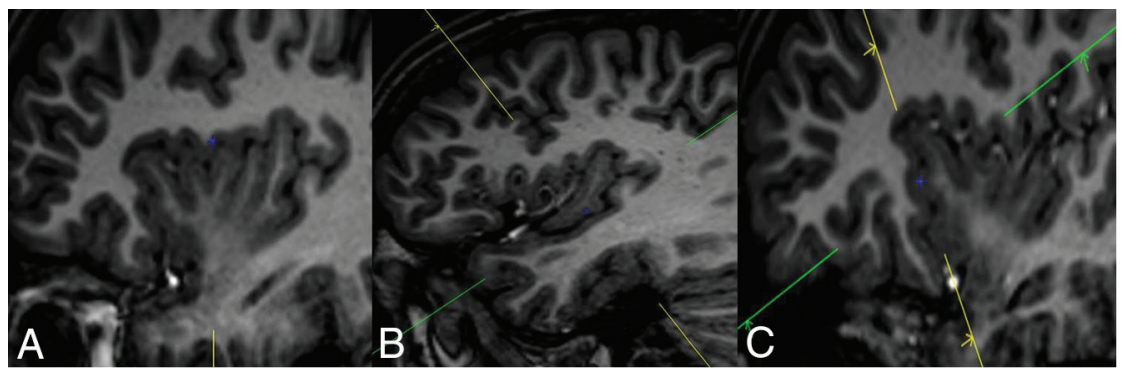

FIG 2. TIWI sagittal view representation of the peri-insular sulci. $A$, Superior peri-insular sulcus. $B$, Inferior peri-insular sulcus. C, Anterior peri-insular sulcus.

The following sequences were analyzed for each patient: 3D T1 turba field echo in the sagittal plane $(\mathrm{TR}=9.7 \mathrm{~ms}, \mathrm{TE}=4.6 \mathrm{~ms}$, matrix $=240 \times 240$, pixel size $=1 \times 1 \mathrm{~mm}, \mathrm{FOV}=235 \times 235$ $\mathrm{mm}$, slice thickness $=1 \mathrm{~mm}$, flip angle $=8^{\circ}$, echo-train length $=$ 159); 3D T2 TSE in the sagittal plane (TR $=2500 \mathrm{~ms}, \mathrm{TE}=252$ ms, matrix $=240 \times 240$, pixel size $=0.7 \times 0.7 \mathrm{~mm}, \mathrm{FOV}=240 \times$ $240 \mathrm{~mm}$, slice thickness $=1 \mathrm{~mm}$, flip angle $=90^{\circ}$, echo-train length $=133$ ).

MRIs were reviewed by 2 experienced neuroradiologists (J.B. and S.K.) working with the knowledge that all patients had insular focal cortical dysplasia (FCD) based on presurgical SEEG and histopathology but blinded to the side and exact location of lesions within the insula. Reviews were first conducted independently, comparing the insulae of both hemispheres in each patient. The results of these reviews were compared. Discordant results were then discussed, and a consensus was reached. A $\kappa$ score was calculated on the basis of the initial independent reviews.

MR imaging studies were reformatted in oblique reference planes to analyze specific structures using an MPR algorithm integrated in the PACS of our institution. The oblique sagittal plane, parallel to the base of the insula, was used to analyze its gyral architecture (Fig 1). Planes parallel and perpendicular to the superior, inferior, and anterior peri-insular sulci were used to analyze the transitional zones between the insular cortex and its neighboring structures (Fig 2). Images were evaluated for signs of
FCD of the insula, the peri-insular sulci, the opercula, or other structures surrounding the insula. These signs included cortical thickening, increased cortical T2WI signal, blurring of the gray/white matter junction, abnormal white matter signal extending from the cortex to the ventricular surface, the transmantle sign ${ }^{5-7}$ and abnormal gyral and/or sulcal patterns, ${ }^{5}$ including shallow sulci or underdeveloped gyri (hypoplasia or a poorly defined gyral pattern), unusually deep sulci or broad gyri (pachygyria), and sulci or gyri with an unusual form (dysmorphic or irregular gyral pattern).

\section{RESULTS}

The clinical and radiologic data are summarized in Tables 1 and 2, respectively.

The 7 patients selected for our study underwent an operation at a mean age of 8.6 years (range, 3-13 years). SEEG was performed in all patients before the operation and demonstrated an ictal onset zone within the insula (anterior insula in 4, posterior insula in 3 ). MR imaging was performed at a maximum of 3 months before the operation and showed subtle abnormalities involving the insula in all patients. One patient had FCD limited to the insula, and 6 had FCD involving the adjacent structures as well.

The most commonly observed MR imaging features of insular FCD in our study were insular and peri-insular blurring, found in 6 of the 7 patients, and an abnormal gyral pattern of the insula, found in all 7 patients. On the basis of these features, 1 radiologist found insular FCDs in all 7 patients, and the other radiologist found insular FCD in 6 of the 7 patients. Neither radiologist found such anomalies in the contralateral insula. The rate of interobserver agreement for the detection of insular dysplasia was almost perfect, with a $\kappa$ score of 0.86 .

The presurgical MR imaging, CT-MR imaging coregistration with SEEG electrodes in place, and postsurgical MR imaging of 2 types of patients are shown: one with an isolated insular lesion (patient 1, Fig 3) and one with an insular lesion extending into the peri-insular sulcus (patient 3, Fig 4).

\section{DISCUSSION}

To our knowledge, this is the first clinical report to describe a method for analyzing MRIs to improve the detection of subtle insular lesions in patients with drug-resistant epilepsy.

In the few studies that have reported insular epilepsy surgery in children, negative findings on MRIs are common, and little attention is given to imaging considerations. ${ }^{4,8,9}$ Lesion identifi- 
Table 1: Clinical data

\begin{tabular}{|c|c|c|c|c|c|c|c|c|c|}
\hline Patient & Sex & $\begin{array}{c}\text { Age at } \\
\text { Seizure } \\
\text { Onset (yr) }\end{array}$ & $\begin{array}{l}\text { Neurologic Status } \\
\text { before Surgery }\end{array}$ & $\begin{array}{c}\text { Age at } \\
\text { Last Surgery } \\
\text { (yr) }\end{array}$ & $\begin{array}{c}\text { Topography of Resective } \\
\text { Surgery }\end{array}$ & $\begin{array}{l}\text { Postsurgical } \\
\text { Deficit }\end{array}$ & Pathology & $\begin{array}{c}\text { FU } \\
\text { Duration } \\
\text { (yr) }\end{array}$ & Engel $^{\mathrm{a}}$ \\
\hline 1 & $M$ & 2.5 & LH, Special education & 13.0 & Posterior Ins and posterior Op & 0 & FCD lla & 4.2 & I \\
\hline 2 & M & 3.2 & $\mathrm{RH}, \mathrm{MoMR}$ & 6.2 & $\begin{array}{l}\text { Anterior Ins and Op, } \\
\quad+\text { frontal Disc }\end{array}$ & $\begin{array}{l}\text { Left facial } \\
\text { paresis }\end{array}$ & FCD lb & 4.2 & I \\
\hline 3 & $\mathrm{~F}$ & 3.5 & $\begin{array}{l}\mathrm{RH}, \text { Normal cognitive } \\
\text { function }\end{array}$ & 14.7 & Posterior Ins and STG & 0 & Negative & 2.4 & I \\
\hline 4 & $\mathrm{~F}$ & 0.8 & $\mathrm{RH}, \mathrm{MoMR}$ & 3.0 & $\begin{array}{l}\text { Anterior Ins+ } \\
\text { frontal Disc }\end{array}$ & 0 & FCD lla & 2.3 & III \\
\hline 5 & M & 0.4 & $\begin{array}{l}\text { Left hemiparesis SMR, } \\
\text { ASD }\end{array}$ & 6.1 & Anterior Ins + frontal Disc & 0 & FCD lla & 0.9 & I \\
\hline 6 & $\mathrm{~F}$ & 2.0 & $\mathrm{LH}, \mathrm{MiMR}$ & 7.6 & $\begin{array}{l}\text { Posterior Ins and parietal Op, } \\
\text { temporal Op }\end{array}$ & 0 & FCD IIb & 3.0 & III \\
\hline 7 & M & 1.0 & LH, MoMR, ASD & 9.7 & Anterior Ins and Inferior Frontal & 0 & FCD $\| b$ & 1.8 & II \\
\hline
\end{tabular}

Note:-LH indicates left-handed; RH, right-handed; MiMR, mild mental retardation; MoMR, moderate mental retardation; SMR, severe mental retardation; ASD, autism spectrum disorder; Op, operculum; Ins, insula; STG, superior temporal gyrus; Disc, disconnection; FU, follow-up; yr, year.

${ }^{a}$ Engel Surgical Outcome Scale.

Table 2: Review of presurgical MRIs in patients with intractable insular epilepsy with no initial detection of insular lesions

\begin{tabular}{|c|c|c|c|c|c|c|c|}
\hline \multirow{2}{*}{$\begin{array}{l}\text { Patient } \\
\text { No. }\end{array}$} & \multirow[b]{2}{*}{ Side } & \multicolumn{2}{|l|}{ Insula } & \multicolumn{2}{|c|}{ Peri-Insular Sulcus } & \multicolumn{2}{|c|}{ Surrounding Structures } \\
\hline & & Gyral Pattern & Blurring & Sulcal Form & Blurring & Gyral Pattern & Blurring \\
\hline 1 & $R$ & Supernumerary ALG & + & - & - & - & - \\
\hline 2 & $\mathrm{R}$ & Poorly defined AL & + & $\begin{array}{l}\text { Irregular anterior portion } \\
\text { of SPS }\end{array}$ & Anterior SPS & - & $\begin{array}{c}\text { Frontal opercula, } \\
\text { orbitofrontal }\end{array}$ \\
\hline 3 & $R$ & Hypoplasia PL & - & - & Posterior IPS & Hypoplasia STG & STG, HG \\
\hline 4 & $\mathrm{R}$ & Irregular AL & + & $\begin{array}{l}\text { Irregular anterior portion } \\
\text { of SPS }\end{array}$ & Anterior SPS & - & - \\
\hline 5 & $\mathrm{R}$ & Irregular AL & + & - & APS & - & $\begin{array}{l}\text { Frontal opercula, } \\
\text { orbitofrontal }\end{array}$ \\
\hline 6 & L & Thick PL & + & - & Posterior IPS & - & HG, temporal stem \\
\hline 7 & L & Poorly defined AL & + & Poorly defined APS & APS & - & Pars orbitalis \\
\hline
\end{tabular}

Note:-ALG indicates the anterior long gyrus; R, right; L, left; SPS, superior peri-insular sulcus; PL, posterior lobule; IPS, inferior peri-insular sulcus; STG, superior temporal gyrus; HG, Heschl gyrus; AL, anterior lobule; APS, anterior peri-insular sulcus; -, normal; +, present; -, absent.

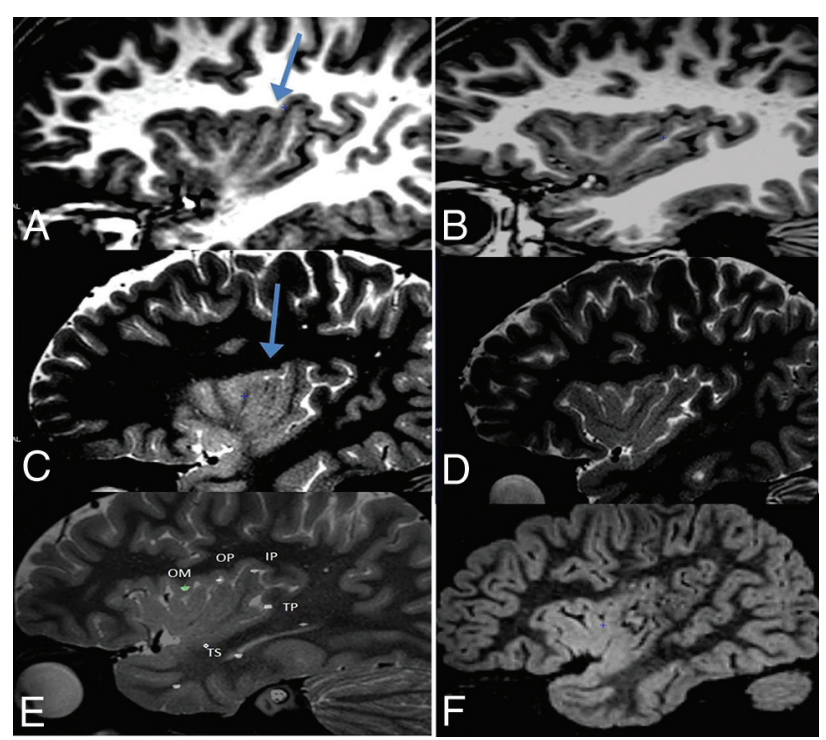

FIG 3. Patient 1. Isolated insular lesion. Oblique sagittal view parallel to the base of the insula. A, TIWI oblique sagittal view shows an unusual gyral pattern of the posterior lobule of the right insula, which shows 3 gyri (arrow). B, TIWI oblique sagittal view shows a normal gyral pattern of the contralateral insula. C, T2WI oblique sagittal view shows blurring of the most anterior long gyrus of the right insula (arrow). D, T2WI oblique sagittal view shows no blurring of the contralateral insula. E, T2WI oblique sagittal view with SEEG electrodes (ictal onset zone around electrode OP). OM, OP, IP, TP, and TS indicate the names of depth electrodes. F, FLAIR sagittal view of the right insula after an operation. cation on MR imaging increases a patient's chances of complete resection, which is an essential prognostic factor of successful epilepsy surgery. ${ }^{10-12}$

In this study, 6 of the 7 detected lesions were classified as FCD on histopathologic analysis according to the Blümcke classification. ${ }^{13}$ The 1 case in which no FCD was found (patient 3 ) may be attributed to the small size of the surgical samples due to the surgical constraints in this region. Those constraints might explain why 2 patients were not seizure-free after the operation (patients 4 and 6).

The MR imaging sequences we chose to analyze are in keeping with the recommended international consensus guidelines for imaging infants and children with recent-onset epilepsy. ${ }^{14}$ Apart from high-resolution 3D-T1WI and 3D-T2WI sequences, there is no consensus on the use of other types of sequences in the clinical setting.

Furthermore, it is necessary to use both T1WI and T2WI sequences to detect FCDs in a young pediatric population because maturation of subcortical white matter appears at different ages on T1WI and T2WI, becoming isointense to cortex at times. ${ }^{15}$ Beyond 24 months, subcortical white matter maturation is usually complete ${ }^{15}$ and FCDs can be detected on both T1WI and T2WI.

The use of MPR postprocessing helped to identify the gyral pattern of the insula and the pattern of the peri-insular sulcus. It also improved the detection of abnormal cortical signal, thickening, and blurring of these structures. All 7 patients were 


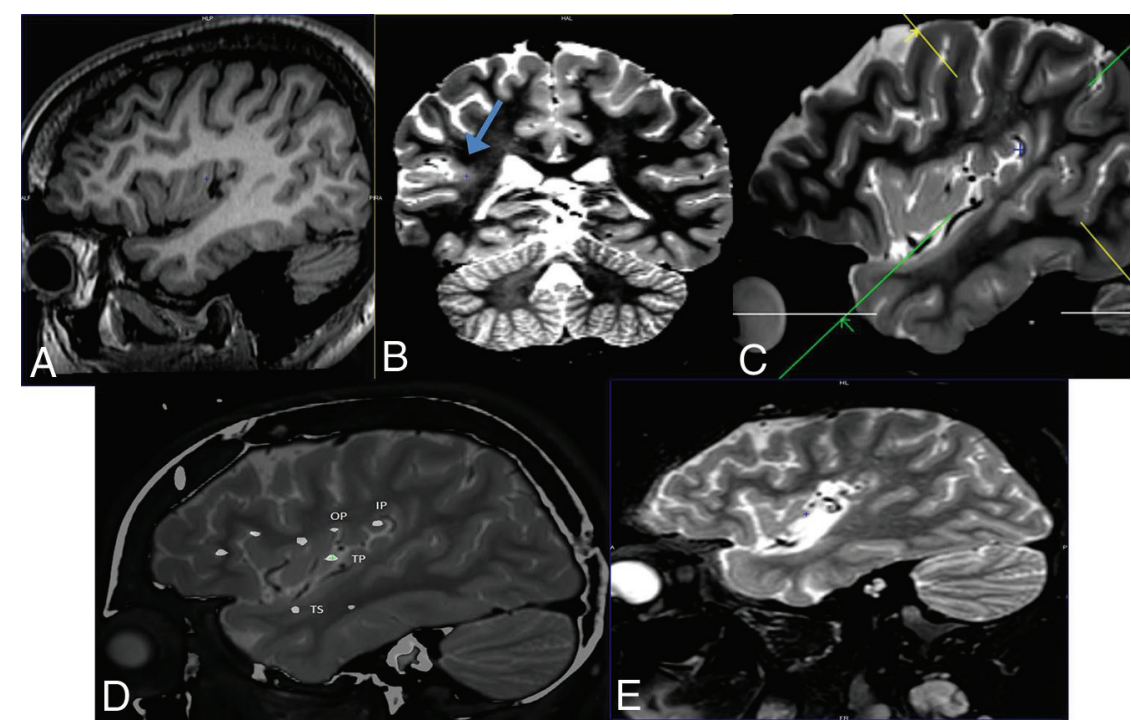

FIG 4. Patient 3. Insular lesion extending into the peri-insular sulcus. $A$, TIWI oblique sagittal view, parallel to the base of the insula, shows hypoplasia of the posterior insular lobule. $B, \mathrm{~T} 2 \mathrm{WI}$ coronal view perpendicular to the inferior peri-insular sulcus shows blurring of the posterior portion of the right inferior peri-insular sulcus (arrow). C, T2WI sagittal view shows the cut plane perpendicular to the posterior portion of the inferior peri-insular sulcus, used to obtain the coronal view in B. D, T2WI sagittal view with SEEG electrodes (ictal onset zone around OP, IP, TP, and TS, representing the depth electrodes). $E, T 2 \mathrm{WI}$ sagittal view after surgical resection of the posterior lobule of the insula and the superior temporal gyrus.

found to have subtle abnormal findings on MR imaging involving the insula, while no such anomaly was detected in the contralateral insula. These findings, along with the strong $\kappa$ score, suggest that the analytic method described in this study is a reliable one, with very few false-positive or false-negative results, while the caveat of a small sample size should be taken into account.

Only patient 1 in our series presented with radiologic signs of FCD limited to the insula itself. Detection of this FCD was made possible through the analysis of the sagittal oblique plane, revealing a posterior lobule with 3 long gyri and blurring of the graywhite matter junction (Fig 3). Literature review of anatomic studies has shown the total number of insular gyri to vary between 4 and 7 (with an average of 5), ${ }^{16-19}$ but no study has shown $>2$ gyri in the posterior lobule of the insula. This unusual gyral pattern in association with localized gray/white matter blurring is suggestive of FCD. Asymmetry of the insular gyral pattern between the 2 hemispheres in the same patient is common ${ }^{19}$ and should not be considered an indication of dysplasia.

In the 6 remaining patients, MR imaging signs suggestive of FCD were found in both the insula and the adjacent peri-insular sulcus, stressing the importance of a systematic analysis of these structures.

All of these lesions, identified retrospectively on presurgical MRIs, were found to be epileptogenic on SEEG recordings. The seizure-onset zones, identified on the intracranial electrodes, were in close proximity to the anomalies identified on MR imaging (Figs 3 and 4), while electrode positioning was guided by electroclinical data before MR imaging detection.

This study has the following limitations: Because refractory insular epilepsy is a rare condition in our experience, a retrospective study design was the most suitable for a preliminary study. As a consequence of the retrospective nature of the study, the parameters of presurgical MRIs were heterogeneous, with insufficient resolution in a large proportion of the examinations, further reducing the number of patients retained in the study. Furthermore, radiologists were blinded to the side and exact location of lesions within the insula and compared both insulae of each patient with each other. This comparison allowed some degree of evaluation of the level of agreement between the 2 radiologists.

The radiologists knowing that all patients had FCD involving the insula may have created a measurement or expectation bias, increasing their tendency to falsely identify lesions. However, this bias did not seem to influence the outcome of the study because no false-positive results were found.

The results of this preliminary study should be confirmed by a multicentric prospective blinded study on a larger population of patients with a control group, allowing a more robust statistical analysis.

\section{CONCLUSIONS}

In this retrospective study, insular lesions were detected in all 7 patients on MRIs initially considered to have negative findings. This highlights the importance of a rigorous imaging technique and analysis by experienced radiologists to reduce the number of so-called "negative" cases.

When exploring refractory epilepsy, the following criteria should be met to optimize the chances of detecting subtle insular lesions on MR imaging: The radiologist must have a good understanding of the gross anatomy of the insula and its surrounding structures. MR imaging sequences must include high-resolution 3D-T1WI and 3DT2WI to detect signal anomalies at various stages of myelination in the young pediatric population. Images should be reconstructed in oblique planes using an MPR algorithm to improve the identification of the gyral pattern of the insula and the pattern of the peri-insular sulcus.

Despite proper analysis of the insular region on presurgical MRI, the full extent of certain forms of FCD still escapes detection. Further advances in the detection of these subtle lesions may be expected in light of the technologic progress being made in MR imaging research using ultra-high-field-strength (7T) scanners to improve spatial resolution as well as the possible contribution of advanced MR imaging techniques such as arterial spin-labeling and diffusion tensor imaging.

\section{REFERENCES}

1. Isnard J, Guénot M, Sindou M, et al. Clinical manifestations of insular lobe seizures: a stereo-electroencephalographic study. Epilepsia 2004;45:1079-90 CrossRef Medline 
2. Nguyen DK, Nguyen DB, Malak R, et al. Revisiting the role of the insula in refractory partial epilepsy. Epilepsia 2009;50:510-20 CrossRef Medline

3. Levitt M, Ojemann J, Kuratani J. Insular epilepsy masquerading as multifocal cortical epilepsy as proven by depth electrode. J Neurosurg Pediatr 2010;5:365-67 CrossRef Medline

4. Dylgjeri S, Taussig D, Chipaux M, et al. Insular and insulo-opercular epilepsy in childhood: an SEEG study. Seizure 2014;23:300-08 CrossRef Medline

5. Colombo N, Tassi L, Galli C, et al. Focal cortical dysplasias: MR imaging, histopathologic, and clinical correlations in surgically treated patients with epilepsy. AJNR Am J Neuroradiol 2003;24: 724-33 Medline

6. Mellerio C, Labeyrie M, Chassoux F, et al. Optimizing MR imaging detection of type 2 focal cortical dysplasia: best criteria for clinical practice. AJNR Am J Neuroradiol 2012;33:1932-38 CrossRef Medline

7. Colombo N, Salamon N, Raybaud C, et al. Imaging of malformations of cortical development. Epileptic Disord 2009;11:194-205 CrossRef Medline

8. Weil A, Le N, Jayakar $\mathrm{P}$, et al. Medically resistant pediatric insular-opercular/periSylvian epilepsy, Part 2: outcome following resective surgery. J Neurosur Pediatr 2016;18:523-35 CrossRef Medline

9. Perry M, Donahue D, Malik S, et al. Magnetic resonance imagingguided laser interstitial thermal therapy as treatment for intractable insular epilepsy in children. J Neurosur Pediatr 2017;20:575-82 CrossRef Medline

10. Hauptman J, Mathern G. Surgical treatment of epilepsy associated with cortical dysplasia: 2012 update. Epilepsia 2012;53(Suppl 4):98 104 CrossRef Medline

11. Rowland N, Englot D, Cage T, et al. A meta-analysis of predictors of seizure freedom in the surgical management of focal cortical dysplasia. J Neurosurg 2012;116:1035-41 CrossRef Medline

12. Englot D, Raygor K, Molinaro A, et al. Factors associated with failed focal neocortical epilepsy surgery. Neurosurgery 2014;75:648-55; discussion 655; quiz 656 CrossRef Medline

13. Blümcke I, Thom M, Aronica E, et al. The clinicopathologic spectrum of focal cortical dysplasias: a consensus classification proposed by an ad hoc Task Force of the ILAE Diagnostic Methods Commission. Epilepsia 2011;52:158-74 CrossRef Medline

14. Gaillard W, Chiron C, Cross J, et al; ILAE, Committee for Neuroimaging, Subcommittee for Pediatric. Guidelines for imaging infants and children with recent-onset epilepsy. Epilepsia 2009;50:2147-53 CrossRef Medline

15. Barkovich AJ. Normal postnatal brain development. In: Barkovich AJ. Pediatric Neuroimaging. 2nd ed. New York: Raven Press; 1995:20-35

16. Türe U, Yaşargil D, Al-Mefty $\mathrm{O}$, et al. Topographic anatomy of the insular region. J Neurosurg 1999;90:720-33 CrossRef Medline

17. Varnavas G, Grand W. The insular cortex: morphological and vascular anatomic characteristics. Neurosurgery 1999;44:127-36; discussion 136-38 CrossRef Medline

18. Augustine J. The insular lobe in primates including humans. Neurol Res 1985;7:2-10 CrossRef Medline

19. Naidich T, Kang E, Fatterpekar G, et al. The insula: anatomic study and MR imaging display at 1.5T. AJNR Am J Neuroradiol 2004;25: 222-32 Medline 